\title{
Spare Capacity Allocation Using Shared Backup Path Protection for Dual Link Failures
}

\author{
Victor Yu Liu* David Tipper ${ }^{\dagger}$ \\ *Huawei Technologies, Boston, Massachusetts, USAＥmail: yuliu@ieee.org \\ ${ }^{\dagger}$ Graduate Telecommunications and Networking Program, University of Pittsburgh, Pittsburgh, Pennsylvania, USA \\ Email: tipper@tele.pitt.edu
}

\begin{abstract}
This paper extends the spare capacity allocation (SCA) problem from single link failure [1] to dual link failures on mesh-like IP or WDM networks. The SCA problem pre-plans traffic flows with mutually disjoint one working and two backup paths using the shared backup path protection (SBPP) scheme. The aggregated spare provision matrix (SPM) is used to capture the spare capacity sharing for dual link failures. Comparing to a previous work by He and Somani [2], this method has better scalability and flexibility. The SCA problem is formulated in a non-linear integer programming model and partitioned into two sequential linear sub-models: one finds all primary backup paths first, and the other finds all secondary backup paths next. The results on five networks show that the network redundancy using dedicated $1+1+1$ is in the range of $313-400 \%$. It drops to 96-181\% in 1:1:1 without loss of dual-link resiliency, but with the trade-off of using the complicated share capacity sharing among backup paths. The hybrid 1+1:1 provides intermediate redundancy ratio at $187-310 \%$ with a moderate complexity. We also compare the passive/active approaches which consider spare capacity sharing after/during the backup path routing process. The active sharing approaches always achieve lower redundancy values than the passive ones. These reduction percentages are about $12 \%$ for $1+1: 1$ and $25 \%$ for $1: 1: 1$ respectively.
\end{abstract}

Index Terms-spare capacity allocation, shared backup path protection, dual link failures, traffic engineering

\section{INTRODUCTION}

Survivability is a critical requirement for reliable services in any network. A variety of survivability techniques (e.g., multiple homing, self-healing rings, pre-planned backup routes, $p$-cycles, etc.) have been proposed for a range of network technologies as surveyed in [3]. The vast majority of the literature and implementations have focused on providing survivability for single link/node/SRLG failures. However, several recent studies have shown the need to address duallink failures in real networks, but this topic has received only sporadic attention in the literature. This study is also initiated from the real dual failure resiliency needs on several backbone networks with high risk of component failures. The approach in this paper is to minimize the pre-planned spare resource while guaranteeing $100 \%$ service recovery upon any duallink failure in mesh-like IP/MPLS or WDM networks. We adopt the shared backup path protection (SBPP) scheme to provide network survivability. The SBPP scheme is an endto-end protection scheme. Each traffic flow will maintain a working path, and in the dual-link failure case two pre-planned backup paths are needed. These three paths must be mutually link disjoint in order to provide dual-link failure survivability. i.e., the traffic can always be restored on all dual link failures, no matter these links fail at the same time or sequentially. If a dual link failure happens and disconnects both the working path and the primary backup path, the secondary backup path can still recover traffic and maintain service continuity. Note that, the spare capacity on links can be shared among backup paths from flows whose working paths are disjoint. This paper will answer two important questions: how to compute this shared spare capacity for dual link failures; and how this could reduce redundancy in the path protection schemes.

The remainder of this section will be a literature review and a quick introduction on the SCA model using SBPP for single failure resiliency. A new integer programming model of the SCA problem for dual-link failures is given in Section II. Based on this model, several backup path protection schemes are described to provide $100 \%$ dual failure resiliency in Section III. Section IV provides the comparative studies and analysis. Lastly, Section V concludes the paper.

\section{A. Related works}

The SBPP scheme has been successfully used to provide end to end path protection for any single link failure. It is one of the basic survivability methods and has received intensive research focus [4], [5], [6]. In particular, we studied the spare capacity allocation problem using the SBPP scheme for any single link/node/SRLG failure in [1]. Here, we extend our previous work to protect against dual-link failures.

In recent years, the dual failure problem has been studied in various directions [7], [8], [9], [10], [11], [12], [13], [14], [15], [16], [17], [2]. Clouqueur and Grover, in [7] studies the performance of a network planned to protect single span failures when dual failures occur. The numerical results show that the majority of the traffic flows will not be impacted by the second failure even though the network is planned for single failure resiliency. Three approaches are further discussed in [8] to study span-restorable design problems for dual failure: minimize cost, maximize restorability, or minimize capacity under a combination of demand restorability requirements. Doucette, et. al, in [9] studies the dual-failure restorability of the SBPP network that is designed to survive all single failures. Doucette and colleagues formulated and studied $p$-cycle network design problems considering dualfailure restorability in [10] and [11]. Choi in [12] proposes a novel method to use a single backup path for each link to 
protect dual failures. It plans the single backup route for each link and any link on this backup route will have a backup route not reusing the originally link. The local protection method by Srini in [13] also uses a single backup path for each link to protect dual-link failure. Backup link mutual exclusion constraints are provided in an ILP model and a heuristic algorithm with polynomial solution time is developed. Zhang in [14] takes a reactive approach to reduce traffic disruption after multiple concurrent failures. A backup reprovisioning is triggered after each failure to protect against the next set of possible failures. A hybrid approach is studied for twolink failure by Ruan in [15]. It pre-plans backup resource to protect the first link failure while using a restoration method for the second. The numerical results indicate a very high restoration ratio for the second failure. This is quite similar to the high redundancy value of the dual-failure restorability on span restorable and SBPP networks in [7] and [9]. For IP over optical networks, various dual failure protection schemes are discussed in [16]. Since both layers have separate protection schemes, it is quite important for backbone network service providers to combine these resource and mechanisms for dual failure resiliency. A recent vendor implementation is Huawei's synergetic protection [17] where the protection schemes on both IP and optical layers are coordinated to achieve the dual failure resiliency with minimum capital expenditure.

In the work closest to ours, He and Somani [2] addresses the joint working and spare capacity allocation problem with an ILP model. In their model, every traffic flow has a set of pre-computed and mutually disjoint candidate paths. These paths will be selected as one working and two backup paths to protect any double link failures. Between any two traffic flows, a set of conditions are listed to indicate when the capacity of two backup paths cannot share on their overlapped links. This is called backup multiplexing. Their ILP model captures all these non-sharing conditions between any two flows so that the roles of candidate backup paths can be chosen to minimize the total capacity. This work contributes to the understanding of spare capacity sharing (or backup multiplexing) for dual link failures. However, there are some limitations in this model. First, it is difficult to scale for large network. The spare capacity sharing (backup multiplexing) constraints in this model depend on complete path information of pair-wise flows. For a large number of flows, the number of the constraints will increase quadratically. Secondly, the pre-computed path set over which the working and backup paths are determined is limited to three disjoint paths in order to reduce the search space of the optimization model. These limitations are part of the reasons to revisit this problem here. We uses an aggregated per-flow information to compute spare capacity sharing for dual failure protection. The same concept has been successfully used for single failure protection in [18], [19], [20]. A detailed literature review on this topic is in [1].

\section{B. SCA Model for Single Failures}

This section briefly reviews the SCA model to protect against single failures on a single layer network. [1], [21]

The network under study uses the Shared Backup Path Protection(SBPP) scheme for any single failures. SBPP allows each flow to use a disjoint backup path to protect its working path upon any single failure. Assume all traffic flows require $100 \%$ restoration for any single failure. Provisioning enough spare capacity becomes critical to such restoration requirement. The notation used in the paper is summarized in Table I. \%begintable[!htbp]

\begin{tabular}{cl} 
& \multicolumn{1}{c}{ TABLE I } \\
& \multicolumn{1}{c}{ NoTATION } \\
\hline \hline$N, L, R, K$ & Numbers of nodes, links, flows and failures \\
$n, l, r, k$ & Indices of nodes, links, flows and failures \\
$\boldsymbol{P}=\left\{\boldsymbol{p}_{r}\right\}=\left\{p_{r l}\right\}$ & Working path link incidence matrix \\
$\boldsymbol{Q}=\left\{\boldsymbol{q}_{r}\right\}=\left\{q_{r l}\right\}$ & Backup path link incidence matrix \\
$\boldsymbol{M}=\operatorname{Diag}\left(\left\{m_{r}\right\}\right)$ & Diagonal matrix of bandwidth $m_{r}$ of flow $r$ \\
$\boldsymbol{G}=\left\{g_{l k}\right\}_{L \times K}$ & Spare provision matrix, $g_{l k}$ is spare capacity \\
$\boldsymbol{G}^{r}=\left\{g_{l k}^{r}\right\}_{L \times K}$ & on link $l$ for failure $k$ \\
$\boldsymbol{s}=\left\{s_{l}\right\}_{L \times 1}$ & Contribution of flow $r$ to $\boldsymbol{G}$ \\
$\boldsymbol{\phi}=\left\{\phi_{l}\right\}_{L \times 1}$ & Spare capacity vector \\
$W_{S} S$ & Spare capacity cost function \\
$\eta=S / W$ & Notal working, spare capacity \\
$o(r), d(r)$ & Origin/destination nodes of flow $r$ \\
$\boldsymbol{B}=\left\{b_{n l}\right\}_{N \times L}$ & Node link incidence matrix \\
$\boldsymbol{D}=\left\{d_{r n}\right\}_{R \times N}$ & Flow node incidence matrix \\
$\boldsymbol{F}=\left\{f_{k l}\right\}_{K \times L}$ & Failure link incidence matrix, $f_{k l}=1$ iff \\
$\boldsymbol{U}=\left\{u_{r k}\right\}_{R \times K}$ & link $l$ fails in failure scenario $k$ \\
& Flow failure incidence matrix, $u_{r k}=1$ \\
$\boldsymbol{T}=\left\{t_{r l}\right\}_{R \times L}$ & iff failure scenario $k$ will affect flow $r$ 's \\
& Forking path \\
\hline \hline & Flow tabu-link matrix, $t_{r l}=1$ iff link $l$ \\
& should not be used on flow $r$ backup path \\
\hline
\end{tabular}

We model an un-capacitated network by a directed graph of $N$ nodes, $L$ links, and $R$ flows. Flow $r, 1 \leq r \leq R$ has its origin/destination node pair $(o(r), d(r))$ and traffic demand $m_{r}$. Working and backup paths of flow $r$ are represented by two $1 \times L$ binary row vectors $\boldsymbol{p}_{r}=\left\{p_{r l}\right\}$ and $\boldsymbol{q}_{r}=\left\{q_{r l}\right\}$ respectively. The $l$-th element in one of the vectors equals to one if and only if (iff) the corresponding path uses link $l$. The path link incidence matrices for working and backup paths are the collections of these vectors, forming two $R \times L$ matrices $\boldsymbol{P}=$ $\left\{p_{r l}\right\}$ and $\boldsymbol{Q}=\left\{q_{r l}\right\}$ respectively. Let $\boldsymbol{M}=\operatorname{Diag}\left(\left\{m_{r}\right\}_{R \times 1}\right)$ denote the diagonal matrix representing demand bandwidth. The topology is represented by the node-link incidence matrix $\boldsymbol{B}=\left(b_{n l}\right)_{N \times L}$ where $b_{n l}=1$ or -1 iff node $n$ is the origin or the destination node of link $l . \boldsymbol{D}=\left(d_{r n}\right)_{R \times N}$ is the flow node incidence matrix where $d_{r n}=1$ or -1 iff $o(r)=n$ or $d(r)=n$. We characterize $K$ failure scenarios in a binary matrix $\boldsymbol{F}=\left\{\boldsymbol{f}_{k}\right\}_{K \times 1}=\left\{f_{k l}\right\}_{K \times L}$. The row vector $\boldsymbol{f}_{k}$ in $\boldsymbol{F}$ is for failure scenario $k$ and its element $f_{k l}$ equals one iff link $l$ fails in scenario $k$. In this way, each failure scenario includes a set of one or more links that will fail simultaneously in the scenario. For a failed node, all its adjacent links are marked as failed. We also denote a flow failure incidence matrix $\boldsymbol{U}=\left\{\boldsymbol{u}_{r}\right\}_{R \times 1}=\left\{u_{r k}\right\}_{R \times K}$, where $u_{r k}=1$ iff flow $r$ will be affected by failure $k$, and $u_{r k}=0$ otherwise. The flow tabu-link matrix $\boldsymbol{T}=\left\{\boldsymbol{t}_{r}\right\}_{R \times 1}=\left\{t_{r l}\right\}_{R \times L}$ has $t_{r l}=1$ iff the backup path of flow $r$ should not use link $l$, and $t_{r l}=0$ otherwise. We can find $\boldsymbol{U}$ and $\boldsymbol{T}$ given $\boldsymbol{P}$ and $\boldsymbol{F}$ as shown in equations (7) and (8) respectively. A binary matrix multiplication operation " $\odot$ " is used in equations (7) and (8). It is a matrix multiply operator that is identical to normal matrix multiplication except that the general numerical 
addition $1+1=2$ will be replaced by the boolean addition $1+1=1$. Using this binary operator, the logical relations among links, paths and failure scenarios are simplified into two matrix operations.

Let $\boldsymbol{G}=\left\{g_{l k}\right\}_{L \times K}$ denote the spare provision matrix (SPM) whose elements $g_{l k}$ are the minimum spare capacity required on link $l$ when failure $k$ occurs. Note that $K=L$ when the SCA protects all single link failures. Given the backup paths $Q$, the demand bandwidth matrix $M$, the working path $\boldsymbol{P}$, and the failure matrix $\boldsymbol{F}, G$ can be determined by equation (3) with the help of (7). The minimum spare capacity required on each link is denoted by the column vector $s=\left\{s_{l}\right\}_{L \times 1}$, which is found in equation (2). The function max in (2) asserts that an element in $s$ is equal to the maximum element in the corresponding row of $\boldsymbol{G}$. It is equivalent to $s \geq \boldsymbol{G}$ in this optimization model. Let $\phi_{l}$ denote the cost function of spare capacity on link $l . \phi=\left\{\phi_{l}\right\}_{L \times 1}$ is a column vector of these cost functions and $\phi(s)$ gives the cost vector of the spare capacities on all links. The total cost of spare capacity in the network is $e^{T} \phi(s)$, where $e$ is unit column vector of size $L$. Here for simplicity, we assume all cost functions $\phi(s)$ are identity functions, i.e., $\phi(s)=s$.

Given the notation and definitions above the spare capacity allocation (SCA) problem can be formulated as follows.

$$
\begin{array}{cc}
\min _{\boldsymbol{Q}, \boldsymbol{s}} & S=\boldsymbol{e}^{T} \boldsymbol{s} \\
\text { s.t. } & \boldsymbol{s}=\max \boldsymbol{G} \\
& \boldsymbol{G}=\boldsymbol{Q}^{T} \boldsymbol{M} \boldsymbol{U} \\
\boldsymbol{T}+\boldsymbol{Q} \leq 1 \\
\boldsymbol{Q} \boldsymbol{B}^{T}=\boldsymbol{D} \\
\boldsymbol{Q}: \text { binary } \\
\boldsymbol{U}=\boldsymbol{P} \odot \boldsymbol{F}^{T} \\
\boldsymbol{T}=\boldsymbol{U} \odot \boldsymbol{F}
\end{array}
$$

This SCA problem has the objective to minimize the total spare capacity in (1) with the constraints (2)-(8). The decision variables are the backup path matrix $Q$ and the spare capacity vector $s$. Constraints (2) and (3) associates these variables, i.e., the spare capacity allocation $s$ is derived from the backup paths in $\boldsymbol{Q}$. Constraint (4) guarantees that every backup path will not use any link which might fail simultaneously with any link on its working path. Flow conservation constraint (5) guarantees that backup paths given in $Q$ are feasible paths of flows in a directed network. Note, the incidence matrices $\boldsymbol{U}$ and $\boldsymbol{T}$ are pre-computed. The matrix $\boldsymbol{U}$ indicates the failure cases that will influence the working paths. The matrix $\boldsymbol{T}$ indicates the links that should be avoided in the backup paths. In this paper, the link load, the traffic flows and their routes are assumed symmetric. In a directed network, each link might have two directions with asymmetric load. In this case, the dimensions of these matrices should be doubled, i.e. $2 L$, instead of $L$. A more detailed explanation of the SCA model above is in [1, eq.(7)-(14)].

The spare provision matrix $\boldsymbol{G}$ can be obtained, in a second approach, by aggregation of per-flow based information. This also contributes significantly on scalability for its dual failure extensions.

First, each flow $r$ has a contribution to $G$ as $\boldsymbol{G}^{r}=$ $\left\{g_{l k}^{r}\right\}_{L \times K}$ in (9), where $\boldsymbol{u}_{r}$ and $\boldsymbol{q}_{r}$ are the $r$-th row vectors in $\boldsymbol{U}$ and $\boldsymbol{Q}$. The spare provision matrix $\boldsymbol{G}$, thus, is calculated in (10). It is also shown in the stack of $G^{r}$ in Fig. 1.

$$
\begin{gathered}
\boldsymbol{G}^{r}=m_{r}\left(\boldsymbol{q}_{r}^{T} \boldsymbol{u}_{r}\right), \quad \forall r, 1 \leq r \leq R \\
\boldsymbol{G}=\sum_{r=1}^{R} \boldsymbol{G}^{r}
\end{gathered}
$$

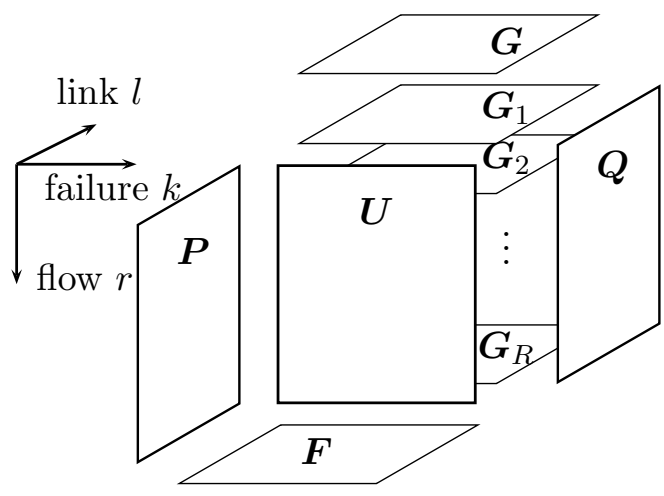

Fig. 1. SCA structure for protecting arbitrary failures

Using above matrices, per-flow based information in $\boldsymbol{P}, \boldsymbol{Q}$ is replaced by $\boldsymbol{G}$ as the stored network state information for spare capacity sharing. The space complexity is reduced from $O(R L)$ to $O(L K)$ and it is independent of the number of flows, $R$. This improves the scalability of the spare capacity sharing information and makes it possible for distributed implementation. Moreover, in this paper we will utilize this feature to capture the spare capacity sharing for dual-link failures.

\section{SCA MODEL FOR DUAL LiNK FAILURES}

This section introduces the spare capacity allocation (SCA) model to protect against dual-link failures. The SBPP scheme is used here. Each flow has one working path and two backup paths. All of which are mutually disjoint so a single duallink failure will only disconnect two paths at the same time. This requires the network to be at least three-connected as is typically assumed in the literature. A recent work by Tsin has provided an easy and fast linear algorithm to verify triconnectivity [22]. In a network with three or more connectivity, at least three disjoint paths can be found using a maximum flow algorithm on a network with every link capacity at one unit and three units of demand from source to destination [23]. It is similar to the algorithm that finds two disjoint paths in [6].

For a network that is not tri-connected, but bi-connected, a set of cut-pairs can be identified to indicate all link pairs whose absence could partition the network. The dual failure cases of these cut-pairs will never be protected. To remedy the requirement of $100 \%$ dual failure resiliency, a work-around resiliency criteria in this situation is a partial dual failure protection method. This is addressed separately in [24]. 


\section{A. Spare Capacity Sharing among Primary Backup Paths}

Before diving into the optimization problem formulation, we show that there is a smaller chance of spare capacity sharing among primary backup paths, comparing to single failures. This comes from Theorem 1 first.

Theorem 1: To protect dual link failures, primary backup paths of any two flows will not be able to share their spare capacity on their overlapped links directly.

Proof: For any two flows, if their primary backup paths overlap on a link, the spare capacity on this link has to be equal to the sum of their bandwidth demand. Because there are dual link failure cases that contain one link in each of the two working paths of these flows, resulting in both primary backup paths being used at the same time. Hence their overlapped link cannot share spare capacity.

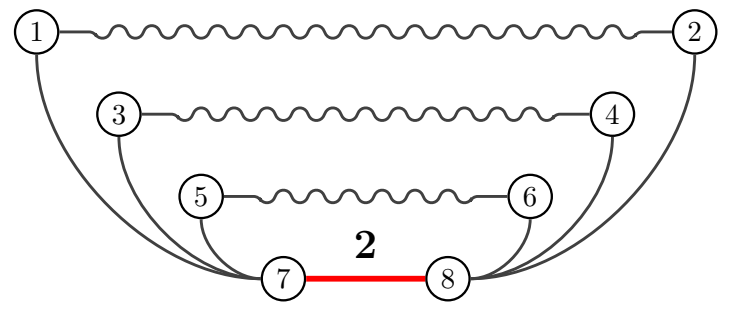

Fig. 2. Two unit spare capacity are shared by three primary backup paths on link $7-8$

In addition, multiple (more than two) primary backup paths might still be able to share their spare capacity. An example is shown in Figure 2. There are three mutually disjoint working paths $1-2,3-4$, and 5-6 in wavy lines, requiring one unit capacity each. Their primary backup paths overlap on link 78 . The spare capacity on this link should be two. This value cannot be one: when a dual failure disconnects two out of three working paths, their primary backup paths will need one unit each on link 7-8. This leads to a minimum capacity of two on link 7-8. Moreover, any dual link failure will not break these three flows at the same time. Their primary backup paths will never fail at the same time. So the spare capacity on link 7-8 does not need to be three, which equals to the spare capacity required for dedicated protection. This example indicates that the spare capacity sharing for the primary backup paths happens only among three or more flows. This makes it difficult to enumerate all these situations for multiple flows. A scalable method of spare capacity sharing should come from every single flow.

\section{B. Collect Information for Each Flow}

As mentioned earlier, the ILP model proposed in [2] focuses on every pair of flows. This limits its scalability. In this paper, a single flow $r$ is put under the spotlight. Its contribution to the spare provision matrix $G^{r}$ is used as the building block for spare capacity sharing. Element $g_{l k}^{r}$ indicates the spare capacity required for flow $r$ on link $l$ when dual-link failure case $k$ happens. The total number of dual link failures is $K=$ $\left(\begin{array}{l}L \\ 2\end{array}\right)$. Each failure $k \in 1 . . K$ stands for a pair of failed links $i, j$ and the index $k$ is determined as $k=(i-1) \times L+(j-i)$ where $1 \leq i<j \leq L$. Assume the failures of two links happen at the same time or shortly close to each other so that the traffic rerouting happens only once. In dual-link failure scenario $k$, the working and backup paths of flow $r$ may be impacted and the spare capacity on their backup paths will need bandwidth reservation as discussed in the following cases:

1) When a dual link failure $k$ breaks the working path, but not the primary backup path, traffic will be protected by the primary backup path. The links on the primary backup path requires a bandwidth demand $m_{r}$.

In the formulation, $p_{r i}=1$ iff link $i$ is on the working path $\boldsymbol{p}_{r}$ and $q_{r j}=1$ iff a link $j$ is on the primary backup path $\boldsymbol{q}_{r}$. Then $p_{r i}\left(1-q_{r j}\right)=1$ indicates link $i$ is on the working path while link $j$ is not on the primary backup path. Let $u_{r k}=p_{r i}\left(1-q_{r j}\right) \oplus\left(1-q_{r i}\right) p_{r j}$, where $\oplus$ is the binary plus which gives $1 \oplus 1=1$. Then $u_{r k}=1$ indicates failure $k$ contains one link on the working path $\boldsymbol{p}_{r}$ but does not contain any link on the primary backup path $\boldsymbol{q}_{r}$. For this failure case $k$, the spare capacity on another link $l$ on the primary backup path should reserve bandwidth $m_{r}$. This is formulated in $g_{l k}^{r[1]}=m_{r} q_{r l} u_{r k}$. These equations can also be rewritten in a vector or matrix format in (11) and (12).

$$
\begin{gathered}
\boldsymbol{G}^{r[1]}=\left\{g_{l k}^{r[1]}\right\}=m_{r} \boldsymbol{q}_{r}^{T} \boldsymbol{u}_{r}^{1} \\
\boldsymbol{u}_{r}^{1}=\operatorname{vec}\left(\boldsymbol{p}_{r}^{T} \overline{\boldsymbol{q}}_{r} \oplus \overline{\boldsymbol{q}}_{r}^{T} \boldsymbol{p}_{r}\right)
\end{gathered}
$$

In the above equations, $\operatorname{vec}(\cdot)$ converts a matrix with index $(i, j)$ into a row vector with index $k$ and $\overline{\boldsymbol{q}}_{r}=$ $\boldsymbol{e}-\boldsymbol{q}_{r}$ where $\boldsymbol{e}$ is a unit row vector with size $L$.

Notice that the length of the row vector $\boldsymbol{u}_{r}^{1}$ is $L^{2}$ instead of number of failures $\left(\begin{array}{l}L \\ 2\end{array}\right)$. This helps to maintain easier matrix formulation and conversion between $k$ and $i, j$. The actual failure size can be easily controlled by removing duplicated cases during the implementation. For this reason, we use $k=(i-1) L+j$ and $K=L^{2}$ in the rest of the paper.

2) When the failure case $k$ contains one link on the working path $\boldsymbol{p}_{r}$ and another link on the primary backup path $\boldsymbol{q}_{r}$, traffic is rerouted to the secondary backup path $\boldsymbol{z}_{r}$. Thus, the links on the secondary backup path need spare capacity to meet bandwidth demand $m_{r}$ for failure case $k$. In this case, $u_{r k}=p_{r i} q_{r j} \oplus q_{r i} p_{r j}$. Then $u_{r k}=1$ indicates failure case $k$ breaks the working path $\boldsymbol{p}_{r}$ and the primary backup path $\boldsymbol{q}_{r}$ at the same time. Hence, the spare capacity on link $l$ on the secondary backup path is $m_{r}$. This gives $g_{l k}^{r[2]}=m_{r} z_{r l} u_{r k}$. These equations can be rewritten in matrix format in (13) and (14).

$$
\begin{gathered}
\boldsymbol{G}^{r[2]}=\left\{g_{l k}^{r[2]}\right\}=m_{r} \boldsymbol{z}_{r}^{T} \boldsymbol{u}_{r}^{2} \\
\boldsymbol{u}_{r}^{2}=\operatorname{vec}\left(\boldsymbol{p}_{r}^{T} \boldsymbol{q}_{r} \oplus \boldsymbol{q}_{r}^{T} \boldsymbol{p}_{r}\right)
\end{gathered}
$$

With two cases above, the final per-flow based spare provision matrix is given in (15).

$$
G^{r}=G^{r[1]}+G^{r[2]} .
$$

The failure matrices $\boldsymbol{F}$ and $\boldsymbol{T}$ in (7) and (8) in the single failure SCA model will not be used in the SCA model for the dual link failures. $\boldsymbol{F}$ becomes the identical matrix for the link failure, and $\boldsymbol{T}$ is simplified to $\boldsymbol{P}$. 


\section{SCA Model for Dual Link Failures}

Using the aggregation of the per-flow based spare provision matrix above, and the additional notation in Table II, the SCA model for dual link failures is formulated in (16)-(22).

TABLE II

ADDITIONAL NOTATION FOR DUAL LINK FAILURES

\begin{tabular}{|c|c|}
\hline $\begin{array}{c}i, j \\
\boldsymbol{Q}=\left\{\boldsymbol{q}_{r}\right\}=\left\{q_{r l}\right\} \\
\boldsymbol{Z}=\left\{\boldsymbol{z}_{r}\right\}=\left\{z_{r l}\right\}\end{array}$ & $\begin{array}{l}\text { Indices of links in a dual failure } k \\
\text { Primary backup path link incidence matrix } \\
\text { Secondary backup path link incidence matrix }\end{array}$ \\
\hline $\boldsymbol{G}^{r[y]}=\left\{g_{l k}^{r[y]}\right\}_{L \times K}$ & $\begin{array}{l}\text { Contribution of flow } r \text { 's } y \text {-th backup path to } \\
\boldsymbol{G}, y=1 \text { or } 2 \text { for primary or secondary }\end{array}$ \\
\hline $\boldsymbol{U}^{\boldsymbol{y}}=\left\{u_{r k}^{y}\right\}_{R \times K}$ & $\begin{array}{l}\text { The incidence matrix for flow's } y \text {-th backup } \\
\text { path and failures, } u_{r k}^{y}=1 \text { iff failure sce- } \\
\text { nario } k \text { will cause flow } r \text { 's traffic to use its } \\
y \text {-th backup path, } y=1 \text { or } 2\end{array}$ \\
\hline$S_{1}, S_{2}$ & $\begin{array}{l}\text { Total spare capacity reserved for the primary } \\
\text { or secondary backup paths }\end{array}$ \\
\hline$\eta_{1}, \eta_{2}$ & $\begin{array}{l}\text { Redundancy value for the primary or sec- } \\
\text { ondary backup paths, } \eta_{y}=S_{y} / W\end{array}$ \\
\hline$\delta_{s}, \gamma_{s}$ & $\begin{array}{l}\text { Value and percentage of redundancy reduc- } \\
\text { tion from the Passive to the Active approach } \\
\text { in scheme } s, s \text { is } 1+1: 1 \text { or } 1: 1: 1\end{array}$ \\
\hline
\end{tabular}

$$
\begin{array}{cc}
\min _{\boldsymbol{Q}, \boldsymbol{Z}, \boldsymbol{s}} & S=\boldsymbol{e}^{T} \boldsymbol{s} \\
\text { s.t. } & \boldsymbol{s}=\max \boldsymbol{G} \\
& \boldsymbol{G}=\boldsymbol{Q}^{T} \boldsymbol{M} \boldsymbol{U}^{\mathbf{1}}+\boldsymbol{Z}^{T} \boldsymbol{M} \boldsymbol{U}^{\mathbf{2}} \\
\boldsymbol{P}+\boldsymbol{Q}+\boldsymbol{Z} \leq 1 \\
\boldsymbol{Q} \boldsymbol{B}^{T}=\boldsymbol{D} \\
\boldsymbol{Z} \boldsymbol{B}^{T}=\boldsymbol{D} \\
\boldsymbol{Q}, \boldsymbol{Z}: \text { binary }
\end{array}
$$

The objective function (16) is very similar to (1), to minimize the total spare capacity. Its decision variables include not only the spare capacity $s$ and the primary backup path matrix $Q$, but also the secondary backup path matrix $Z$. In constraint (17), the spare capacity column vector $s$ is derived from the maximum values of elements in rows, across all failures, in the spare provision matrix $G$. It indicates that the required spare capacity on a link is equivalent to the highest "watermark" from all possible dual link failures. In constraint (18), the spare provision matrix is derived from backup paths. It is a matrix format, equivalent to the aggregation of per-flow based information from (10), (15), (11), and (13). Constraint (19) requires the working and backup paths to be mutually disjoint, i.e., these paths will use the same link at most once. Constraints (20) and (21) are the flow balance requirements, to guarantee these paths in $Q$ and $Z$ to be valid routes between the source and destination nodes. Constraint (22) requires backup path decision variables to be binary ensuring each backup path is not bifurcated. Row vectors $\boldsymbol{u}^{y}$ in $\boldsymbol{U}^{y}, y \in 1,2$ are derived in (12) and (14) to indicate which failure case $k$ could cause traffic detour to its primary or secondary backup path.

\section{Solution Approach}

The SCA model above is a non-linear integer programming problem. Both terms in the right side of (18) involve two design variables to multiply together. In the first term $\boldsymbol{Q}^{T} \boldsymbol{M} \boldsymbol{U}^{\mathbf{1}}$, the design variable $\boldsymbol{Q}$ times another variable $\boldsymbol{U}^{\mathbf{1}}$ which is further computed from $\boldsymbol{q}_{r}$ in (12). Similarly, the second term $\boldsymbol{Z}^{T} \boldsymbol{M} \boldsymbol{U}^{2}$ is also based on the multiplication of decision variables $\boldsymbol{Z}$ and $\boldsymbol{Q}$ via $\boldsymbol{u}^{2}$ in (14). To remove the non-linearity in the first term, we replace (12) with a slightly different equation (23), where the original $\overline{\boldsymbol{q}}_{r}$ is replaced by $\boldsymbol{e}$.

$$
\boldsymbol{u}_{r}^{1}=\operatorname{vec}\left(\boldsymbol{p}_{r}^{T} \boldsymbol{e} \oplus \boldsymbol{e}^{T} \boldsymbol{p}_{r}\right)
$$

This change increases the SPM value on the links along the primary backup path, under the failure cases that also disconnect this primary backup path. These values are zero in the previous equation (12). Consequently, these values in the spare provision matrix might slightly decrease the spare capacity sharing chances with other flows.

To cope with the non-linearity in the second term, we partition the original SCA model into two models: one is to find the primary backup paths first, and the other is to find the secondary backup paths afterward. It simplifies the solution approach but might increase the required spare capacity. It is possible to replace the non-linear constraint by adding extra variables and constraints using integer programming modeling techniques. It might achieve better optimality. However, this paper takes the partitioning way for better computation speed.

1) Find Primary Backup Paths First: In order to find the primary backup paths, the ILP model is given in (24)-(29) to find total spare capacity for primary backup paths $S_{1}$.

$$
\begin{array}{cc}
\min _{\boldsymbol{Q}, \boldsymbol{s}_{1}} & S_{1}=\boldsymbol{e}^{T} \boldsymbol{s}_{1} \\
\text { s.t. } & \boldsymbol{s}_{1}=\max \boldsymbol{G}^{[1]} \\
& \boldsymbol{G}^{[1]}=\boldsymbol{Q}^{T} \boldsymbol{M} \boldsymbol{U}^{\mathbf{1}} \\
& \boldsymbol{P}+\boldsymbol{Q} \leq 1 \\
& \boldsymbol{Q} \boldsymbol{B}^{T}=\boldsymbol{D} \\
& \boldsymbol{Q}: \text { binary }
\end{array}
$$

Most of these equations are similar to the previous model except the incidence matrix $\boldsymbol{U}^{1}$ is found using (23).

2) Find Secondary Backup Paths with Fixed Primary Backup Paths: After the primary backup paths are determined, the secondary backup paths can be determined with the objective function to minimize the total spare capacity in (16) with the design variables $Z$ and $s$. The constraints are (17)(22), (12), and (14). However, the difference here is that the primary backup paths $\boldsymbol{Q}$ and its derived matrix $\boldsymbol{U}^{[1]}$ have been fixed. The formulation becomes an ILP model.

Both of these ILP models are NP-complete. We will solve them on smaller networks using commercial software AMPL with ILP solvers, such as CPLEX or Gurobi, that normally use the branch and cut algorithm. To solve for larger networks, a previous heuristic algorithm, called successive survivable routing (SSR), can be extended to find near optimal solutions quickly. This is left for future work.

\section{Backup Path Protection Schemes}

The backup path protection schemes can be coded on how the backup paths reserve spare capacity: " +1 " for dedicated 
spare capacity and ": 1 " for shared spare capacity. ${ }^{1}$

$1+1+1$ stands for the dedicated path protection with no capacity sharing among backups.

$1+1: 1$ is the hybrid backup path protection with the dedicated spare capacity for primary backup paths and shared bandwidth for secondary backup paths.

1:1:1 is the SBPP scheme that allows spare capacity sharing among all backup paths.

We further study the relationship between the spare capacity sharing and the backup path routing for $1+1: 1$ and $1: 1: 1$ schemes using two methods: active and passive. Active method combines the spare capacity sharing during the backup path routing. Passive method will find the backup paths first, then determines the shared spare capacity. Based on these definitions, five schemes for dual link failure protection are compared in terms of the total spare capacity for primary backup paths $S_{1}$, and for secondary backup paths $S_{2}$, where $S_{1}, S$ are found in (24),(16) and $S_{2}=S-S_{1}$. We also listed three schemes for single link failures [1].

1) Dedicated $1+1+1$ is the dedicated path protection scheme. It uses the shortest path algorithm to find mutually disjoint primary and secondary backup paths. The spare capacity on these backup paths are reserved separately. There is no spare capacity sharing. This scheme should use the largest total spare capacity.

2) Passive $1+1: 1$ also uses the shortest path algorithm to find mutually disjoint primary and secondary backup paths. Only after all backup paths are found, the spare capacity among secondary backup paths will then be shared based on the spare provision matrix method in (30) and (31).

$$
\begin{gathered}
\boldsymbol{s}_{2}=\max \boldsymbol{G}^{[2]} \\
\boldsymbol{G}^{[2]}=\boldsymbol{Z}^{T} \boldsymbol{M} \boldsymbol{U}^{\mathbf{2}}
\end{gathered}
$$

3) Active $1+1: 1$ scheme uses the shortest path algorithm to find mutually disjoint primary backup paths. After that, the ILP model in $§$ II-D2 is used to find the secondary backup paths.

4) Passive 1:1:1 scheme uses the shortest path algorithm to find mutually disjoint primary and secondary backup paths. After these paths are found, their spare capacity for all backup paths will then be shared using the spare provision matrix method in (17) and (18).

5) Active 1:1:1 scheme considers spare capacity sharing during the process of finding both backup paths. We use the ILP models sequentially in $\S$ II-D1 and $\S I I-D 2$ to find these backup paths which also produce the minimum total spare capacity in these ILP sub-models. For large networks, finding approximation solutions is currently under development.

6) Dedicated $1+1$ is the dedicated path protection scheme for single link failure. It uses the shortest path algorithm to find mutually disjoint backup paths and the spare

\footnotetext{
${ }^{1}$ This could be defined as " $1+$ " or " 1 :" for dedicated/shared spare capacity. It will be compatible with the "M:N" protection where M backup links protect $\mathrm{N}$ working links. For this reason, we do not use aggregated form such as "1:2" or " $2: 1$ " to avoid confusion.
}

capacity on these backup paths are reserved dedicated to this flow. There is no sharing.

7) Passive 1:1 scheme uses the shortest path algorithm to find a backup path disjoint from its working path. The spare capacity for all backup paths will then be shared using the spare provision matrix method in (2) and (3).

8) Active $1: 1$ scheme considers spare capacity sharing during the process of finding both backup paths. The ILP model in $\S I-B$ or [1] is used to find these backup paths which also produce the minimum total spare capacity.

\section{NUMERICAL STUDY}

Numerical experiments were performed on the five network topologies shown in Figure 3. The results are given in Table III. The network node count ranges from 5 to 50 with an average node degree from 3.4 to 4.4 , as shown in the first five rows. Each network has a full-meshed flows with one unit bandwidth demand. The working paths are pre-determined using the shortest path algorithm. The total working capacity $W$ is listed in the sixth row.

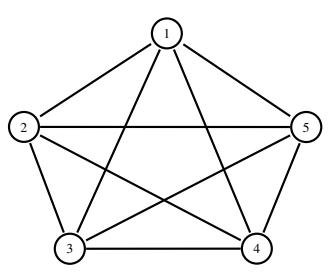

Net1: $(5,10,20)$

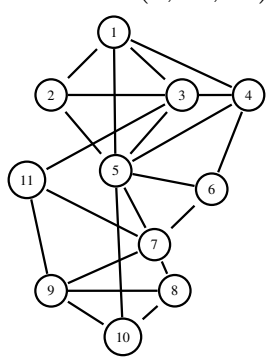

Net3: $(11,22,110)$

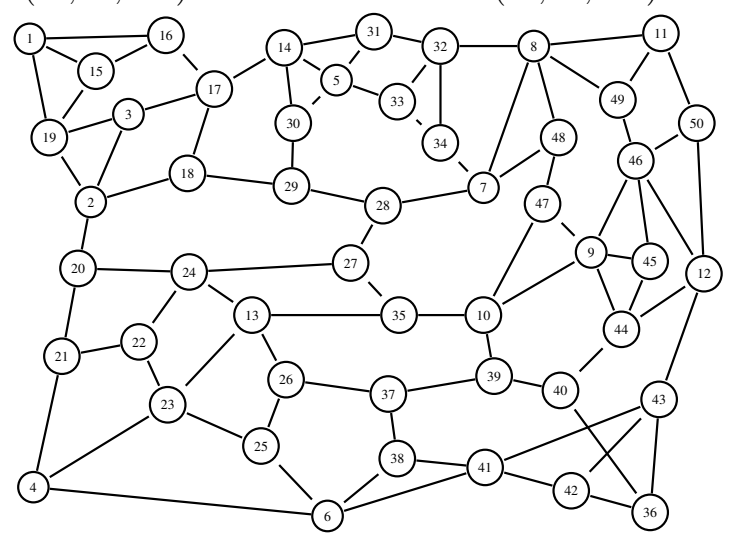

Net5: $(N, L, R)=(50,86,2450)$

Fig. 3. Networks used for numerical study

The following rows list the numerical results for the different path protection schemes. We focus on the total spare capacity for primary, secondary, and both backup paths, i.e., 
TABLE III

RESULTS ON FIVE NETWORKS

\begin{tabular}{|c|c|c|c|c|c|c|}
\hline & Network & $\overline{1}$ & $\overline{\overline{2}}$ & $\overline{\overline{3}}$ & $\overline{\overline{4}}$ & $\overline{\overline{5}}$ \\
\hline Num. & $\operatorname{nodes} N$ & 5 & 10 & 11 & 17 & 50 \\
\hline Num & f links $L$ & 10 & 22 & 22 & 33 & 86 \\
\hline Avg. & le deg. $d$ & 4 & 4.4 & 4 & 3.9 & 3.44 \\
\hline Num. & flows $R$ & 20 & 90 & 110 & 272 & 2450 \\
\hline Total work & cap. $W$ & 20 & 142 & 190 & 626 & 10888 \\
\hline & $\overline{S_{1}}$ & $\overline{40}$ & $\overline{198}$ & $\overline{282}$ & $\overline{846}$ & 15136 \\
\hline & $S_{2}$ & 40 & 246 & 376 & 1216 & 22208 \\
\hline Dedicated & $S$ & 80 & 444 & 658 & 2062 & 37344 \\
\hline $1+1+1$ & $\eta_{1}$ & $200 \%$ & $139 \%$ & $148 \%$ & $135 \%$ & $139 \%$ \\
\hline & $\eta_{2}$ & $200 \%$ & $173 \%$ & $198 \%$ & $194 \%$ & $204 \%$ \\
\hline & $\eta$ & $400 \%$ & $313 \%$ & $346 \%$ & $329 \%$ & $343 \%$ \\
\hline & $\overline{\overline{S_{1}}}$ & $\overline{\overline{40}}$ & $\overline{198}$ & 282 & $\overline{846}$ & $\bar{~} 15136$ \\
\hline & $S_{2}$ & 22 & 110 & 192 & 494 & 8938 \\
\hline Passive & $S$ & 62 & 308 & 474 & 1340 & 24074 \\
\hline $1+1: 1$ & $\eta_{1}$ & $200 \%$ & $139 \%$ & $148 \%$ & $135 \%$ & $139 \%$ \\
\hline & $\eta_{2}$ & $110 \%$ & $77 \%$ & $101 \%$ & $79 \%$ & $82 \%$ \\
\hline & $\eta$ & $310 \%$ & $217 \%$ & $249 \%$ & $214 \%$ & $221 \%$ \\
\hline & $S_{1}$ & 40 & 198 & 282 & 846 & 15136 \\
\hline & $S_{2}$ & 14 & 74 & 142 & 322 & - \\
\hline Active & $S$ & 54 & 272 & 424 & 1168 & $-\ddagger$ \\
\hline $1+1: 1$ & $\eta_{1}$ & $200 \%$ & $139 \%$ & $148 \%$ & $135 \%$ & $139 \%$ \\
\hline & $\eta_{2}$ & $70 \%$ & $52 \%$ & $75 \%$ & $51 \%$ & - \\
\hline & $\eta$ & $270 \%$ & $192 \%$ & $223 \%$ & $187 \%$ & - \\
\hline & $\delta_{1+1: 1}$ & $40 \%$ & $25 \%$ & $26 \%$ & $27 \%$ & - \\
\hline & $\gamma_{1+1: 1}$ & $13 \%$ & $12 \%$ & $11 \%$ & $13 \%$ & - \\
\hline & $\overline{S_{1}}$ & $\overline{24}$ & $\overline{152}$ & $\overline{216}$ & $\overline{588}$ & 10450 \\
\hline & $S_{2}$ & 12 & 44 & 128 & 220 & 6494 \\
\hline Passive & $S$ & 36 & 196 & 344 & 808 & 16944 \\
\hline $1: 1: 1$ & $\eta_{1}$ & $120 \%$ & $107 \%$ & $114 \%$ & $94 \%$ & $96 \%$ \\
\hline & $\eta_{2}$ & $60 \%$ & $31 \%$ & $67 \%$ & $35 \%$ & $60 \%$ \\
\hline & $\eta$ & $180 \%$ & $138 \%$ & $181 \%$ & $129 \%$ & $156 \%$ \\
\hline & $S_{1}$ & 20 & 92 & 168 & $390 \dagger$ & $-f$ \\
\hline & Time(s) & 6.2 & 598 & 19 & $20 \mathrm{hr} \dagger$ & - \\
\hline & $S_{2}$ & 16 & 56 & 96 & 214 & - \\
\hline & $S$ & 36 & 148 & 264 & 604 & - \\
\hline Active & Time(s) & 0.27 & 1.2 & 0.42 & 3.0 & - \\
\hline $1: 1: 1$ & $\eta_{1}$ & $100 \%$ & $65 \%$ & $88 \%$ & $62 \%$ & - \\
\hline & $\eta_{2}$ & $80 \%$ & $39 \%$ & $51 \%$ & $34 \%$ & - \\
\hline & $\eta$ & $180 \%$ & $104 \%$ & $139 \%$ & $96 \%$ & - \\
\hline & $\delta_{1: 1: 1}$ & $0 \%$ & $34 \%$ & $43 \%$ & $32 \%$ & - \\
\hline & $\gamma_{1: 1: 1}$ & $0 \%$ & $24 \%$ & $24 \%$ & $25 \%$ & - \\
\hline$\overline{\overline{\text { Passive }}}$ & $\overline{S_{1}}$ & $\overline{14}$ & $\overline{\overline{94}}$ & $\overline{142}$ & $\overline{\overline{380}}$ & $\overline{6738}$ \\
\hline $1: 1$ & $\eta_{1}$ & $70 \%$ & $66 \%$ & $75 \%$ & $61 \%$ & $62 \%$ \\
\hline & $S_{1}$ & 10 & 50 & 94 & 208 & 4544 \\
\hline Active & Time(s) & 2 & 11 & 11 & 713 & $16.6 \mathrm{hr}$ \\
\hline $1: 1$ & $\eta_{1}$ & $50 \%$ & $35 \%$ & $49 \%$ & $33 \%$ & $42 \%$ \\
\hline & $\delta_{1 \cdot 1}$ & $20 \%$ & $31 \%$ & $25 \%$ & $27 \%$ & $20 \%$ \\
\hline & $\gamma_{1: 1}$ & $40 \%$ & $88 \%$ & $51 \%$ & $83 \%$ & $48 \%$ \\
\hline
\end{tabular}

$\uparrow:$ This is a near optimal solution with an absolute MIP gap of 4 using Gurobi after 20 hours. Other ILP results are optimal using CPLEX. Time is in second on an Intel Core i5 $2.4 \mathrm{GHz}$ CPU. ‡: No solution found due to memory limitation in AMPL. interesting observations are noted as follows:

The redundancy can be reduced by increasing the complexity. The $1+1+1$ dedicated protection scheme has the highest redundancy range of $313-400 \%$. It reduces down to $96-181 \%$ in 1:1:1 SBPP scheme without loss of dual-link resiliency. This trade-off between redundancy and complexity is very important to evaluate various protection schemes. The hybrid 1+1:1 scheme provides intermediate redundancy ratio at $187-310 \%$, while its complexity is also moderate between the other two schemes.

The active approach always has lower redundancy than the passive approach. The results show that active scheme always has lower redundancy compared to corresponding passive scheme in both $1+1: 1$ and $1: 1: 1$ schemes. Furthermore, we denote the reduction value $\delta_{s}$ and ratio $\gamma_{s}$ for the network redundancy from the Passive to Active approach in the same schemes $s$, and compute them in (32).

$$
\begin{aligned}
\delta_{s} & =\eta_{s}^{\text {Passive }}-\eta_{s}^{\text {Active }} \\
\gamma_{s} & =\delta_{s} / \eta_{s}^{\text {Passive }}, s \in\{1+1: 1,1: 1: 1,1: 1\}
\end{aligned}
$$

from the numerical results in Table III, we notice that $\gamma_{1+1: 1}$ is about $12 \%$ across four networks, while $\gamma_{1: 1: 1}$ is about $25 \%$ for the three non-trivial networks $2-4$. This is a very interesting findings and needs further analysis.

The secondary backup paths use less spare capacity than the primary backup paths in SBPP. In both 1+1:1 and 1:1:1 schemes, the secondary backup paths uses less spare capacity than that of the primary backup paths need. The ratio of $\frac{S_{2}}{S_{1}}$ varies from 0.8 in the result of active $1: 1: 1$ on network 1 down to 0.29 in the result of passive $1: 1: 1$ on network 2 . On the contrary in the dedicated $1+1+1$ scheme, the secondary backup paths use same or more spare capacity than the primary backup paths with the same ratio varies from 1 to 1.47 .

It requires much more redundancy to protect $100 \%$ dual link failures than single link failures. When we compare the network redundancy on the first four networks, the Active $1: 1: 1$ has about 1.8-2.6 times of redundancy value comparing to that of the Active 1:1 scheme. This large increase on the redundancy is critical for the network service providers to make decision between these protection mechanisms and their extra resource requirements.

\section{CONCLUSION}

This paper answers two important questions for dual link failure protection: how to compute the spare capacity sharing in SBPP; and how it helps reducing network redundancy in the backup path protection schemes. Numerical study shows that the five backup path protection schemes cover a spectrum of network redundancy in a decreasing order, while their sharing complexity increases.

\section{ACKNOWLEDGEMENT}

We would like to thank anonymous reviewers and Dr. Wenda Ni for their valuable comments. stack these values together so the total redundancy $\eta$ can be seen by the top of the bar. From the numerical results several
$S_{1}, S_{2}, S$ respectively. Their redundancy value $\eta_{1}, \eta_{2}, \eta$ are also listed, where $\eta=\frac{S}{W}$. For the "Active 1:1:1", the second) to compute $S_{1}$ and $S$ using AMPL with CP. the computation time is less than seconds hence ignored

In the bottom of Table III, the results from the $1: 1$ scheme dual link failures.

These redundancy values $\eta_{1}$ and $\eta_{2}$ in the results table are also drawn as a bar chart in Figure 4. For each scheme, we 


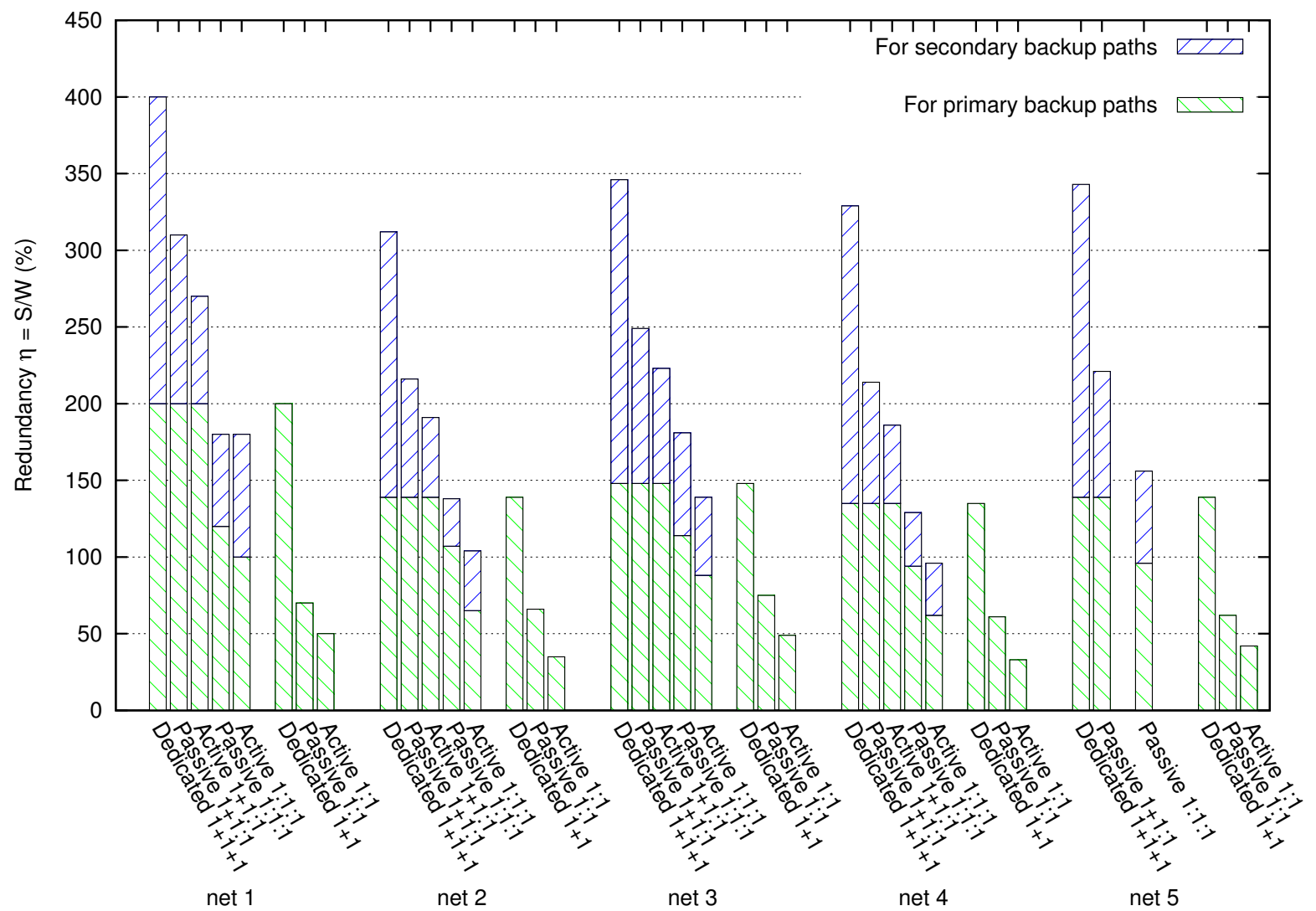

Fig. 4. Comparison of Redundancy $\eta=\frac{S}{W}$ among path protection schemes.

\section{REFERENCES}

[1] Y. Liu, D. Tipper, and P. Siripongwutikorn, "Approximating optimal spare capacity allocation by successive survivable routing," IEEE/ACM Transactions on Networking, vol. 13, no. 1, pp. 198-211, Feb. 2005.

[2] W. He and A. K. Somani, "Path-based protection for surviving doublelink failures in mesh-restorable optical networks," in Proceeding of IEEE GLOBECOM, 2003, pp. 2258-2563.

[3] Y. Qian, J. Joshi, D. Tipper, and P. Krishnamurthy, Information Assurance: Dependability and Security in Networked Systems. Morgan Kauffman Publishers, 2008.

[4] W. Grover, Mesh-based Survivable Networks: Options and Strategies for Optical, MPLS, and ATM Networking. Prentice Hall PTR, 2003.

[5] M. Pióro and D. Medhi, Routing, Flow and Capacity Design in Communication and Computer Networks. Morgan Kauffman, 2004.

[6] R. Bhandari, Survivable Networks Algorithms for Diverse Routing. Kluwer Academic Publishers, 1999.

[7] M. Clouqueur and W. D. Grover, "Availability analysis of span-restorable mesh networks," IEEE Journal on Selected Areas of Communications, vol. 20, no. 4, pp. 810-821, May 2002.

[8] — , "Mesh-restorable networks with complete dual-failure restorability and with selectively enhanced dual-failure restorability properties," in Proceedings of Optical Networking and Communications Conference (OptiComm 2002), SPIE vol. 4874, Boston, MA, Jul. 2002, pp. 1-12.

[9] J. Doucette, M. Clouqueur, and W. D. Grover, "On the availability and capacity requirements of shared backup path-protected mesh networks," Optical Networks Magazine, Special Issue on Engineering the Next Generation Optical Internet, vol. 4, no. 6, pp. 29-44, Nov./Dec. 2003.

[10] J. Doucette, W. Li, and M. Zuo, "Failure-specific p-cycle network dualfailure restorability design," in Intl. Workshop on Design of Reliable Communication Networks (DRCN), La Rochelle, France, Oct. 2007.

[11] W. Li, J. Doucette, and M. Zuo, "p-cycle network design for specified minimum dual-failure restorability," in Proceeding of IEEE International Conference on Communications, Jun. 2007.

[12] H. Choi, S. Subramaniam, and H.-A. Choi, "Loopback recovery from double-link failures in optical mesh networks," IEEE/ACM Transactions on Networking, vol. 12, no. 6, pp. 1119-1130, Dec. 2004.

[13] S. Ramasubramanian and A. Chandak, "Dual-link failure resiliency through backup link mutual exclusion," IEEE/ACM Transactions on Networking, vol. 16, no. 1, pp. 157-169, Feb. 2008.

[14] J. Zhang, K. Zhu, N. S. Matloff, and B. Mukherjee, "Backup reprovisioning to remedy the effect of multiple link failures in WDM mesh networks," IEEE Journal on Selected Areas of Communications, vol. 24 no. 8, pp. 57-67, Aug. 2006.

[15] L. Ruan and T. Feng, "A hybrid protection/restoration scheme for two-link failure in WDM mesh networks," in Proceeding of IEEE GLOBECOM, Miami, FL, 2010.

[16] R. Prinz, A. Autenrieth, and D. Schupke, "Dual failure protection in multilayer networks based on overlay or augmented model," in Intl. Workshop on Design of Reliable Communication Networks (DRCN), Island of Ischia, Naples, Italy, Oct. 16-19 2005.

[17] Q. Yang, "Bearer network-synergetic protection for IP and optical bearer networks," Huawei Communicate, no. 58, pp. 31-34, Dec. 2010.

[18] C. Dovrolis and P. Ramanathan, "Resource aggregation for fault tolerance in integrated service networks," ACM Computer Communication Review, vol. 28, no. 2, pp. 39-53, 1998.

[19] D. Medhi and D. Tipper, "Some approaches to solving a multi-hour broadband network capacity design problem with single-path routing," Telecommunication Systems, vol. 13, no. 2, pp. 269-291, 2000.

[20] M. Kodialam and T. Lakshman, "Dynamic routing of bandwidth guaranteed tunnels with restoration," in Proceeding of IEEE INFOCOM, 2000

[21] Y. Liu, D. Tipper, and K. Vajanapoom, "Spare capacity allocation in twolayer networks," IEEE Journal on Selected Areas of Communications, vol. 25, no. 5, pp. 974-986, Jun. 2007.

[22] T. H. Tsin, "Yet another optimal algorithm for 3-edge-connectivity," Journal of Discrete Algorithms, vol. 7, pp. 130-146, 2009.

[23] R. K. Ahuja, T. L. Magnanti, and J. B. Orlin, Network Flows: Theory, Algorithms and Applications. New York: Prentice Hall, 1993.

[24] V. Y. Liu, Partial Dual Link Failure Protection on Bi-Connected Networks, Huawei Technologies, 2011, technical report. 